\title{
Entanglement versus cooling in the system of a driven pair of two-level qubits longitudinally coupled with a boson mode field
}

\author{
Elena Cecoi, ${ }^{1}$ Viorel Ciornea, ${ }^{1}$ Aurelian Isar, ${ }^{2}$ and Mihai A. Macovei ${ }^{1, *}$ \\ ${ }^{1}$ Institute of Applied Physics, Academiei str. 5, MD-2028 Chişinău, Moldova \\ ${ }^{2}$ Horia Hulubei National Institute of Physics and Nuclear Engineering, \\ Reactorului str. 30, MG-6 Bucharest - Măgurele, Romania
}

(Dated: July 29, 2019)

\begin{abstract}
The relationship among the entanglement creation within coherently pumped and closely spaced two-level emitters longitudinally coupled with a single-mode boson field, and the subsequent quantum cooling of the boson mode is investigated. Even though the two-level qubits are resonantly driven, we have demonstrated an efficient cooling mechanism well below limits imposed by the thermal background. Furthermore, the cooling effect is accompanied by entanglement of the qubit pair components when the dipole-dipole frequency shift is close to the frequency of the boson mode. The maximum boson mode cooling efficiency realizes on the expense of the entanglement creation. Importantly, this occurs for rather weak external pumping fields protecting the sample from the deteriorations. Finally, the conditions to effectively optimize these effects are described as well.
\end{abstract}

*Electronic address: macovei@phys.asm.md 


\section{INTRODUCTION}

Entanglement in a few-atom system attracted enormous attention over last few decades [1 8]. Small qubit samples may form buildings blocks for even larger networks with huge potential applications for quantum technologies [9-19]. Generally, a thorough description of the entanglement creation in a two-atom system was given in [20]. From this point of view artificial atomic systems have been widely investigated as well. Particularly, experimental realization of entanglement in two coupled charge qubits was performed in [21]. Entanglement of two quantum dots inside a cavity injected with squeezed vacuum was predicted as well, in [22]. Ultra-strong dipole-dipole interacting two-level superconducting flux qubits are naturally entangled through their corresponding environmental reservoir and maximum coherence can be induced too [23]. Furthermore, a pair of moderately dipole-dipole coupled and laser pumped two-level quantum dots get maximal entangled via their environmental phonon thermostat which facilitates also the creation of a subradiant two-qubit state [24]. Recently, based on quantum dots systems, a relevant experimental realization of an interconnection among two qubits located five meters apart from each other, via single photons, was reported in Ref. [25].

Often to realize quantum states of matter or light one requires ground-state cooled individual or coupled quantum systems, respectively. In this context, cooling of a quantum circuit via coupling to an independent or Dicke-like interacting multiqubit ensemble was demonstrated in [26]. The cooling of a nanomechanical resonator coupled to two interacting flux qubits via the corresponding subradiant Dicke states was demonstrated as well, in Ref. [27]. A scheme for ground-state cooling of a mechanical resonator coupled to two coupled quantum dots forming an effective $\Lambda$-type three-level structure was presented in [28]. Ground-state cooling of a nanomechanical oscillator with $N$ spins was recently proposed in [29].

Evidently, there is a considerable effort done to elucidate the relationship among the entanglement and cooling phenomena. For instance, it was found that entanglement enhances cooling in microscopic quantum refrigerators [30]. The atom-membrane cooling and entanglement using cavity electromagnetically induced transparency was investigated in Ref. [31]. Ground-state cooling enabled by critical coupling and dark entangled states was found in [32]. Further interesting works on cooling or entanglement processes are presented in 
Refs. [33 39].

Here we shall take the opportunity of these advances and investigate the interconnection of the entanglement process in a coherently and resonantly pumped dipole-dipole interacting two-qubit system, and the cooling effects of a boson mode with whom the qubits are longitudinally coupled. Both the quantum subsystems are damped via their corresponding environmental reservoirs. We have found that the cooling of the boson mode is accompanied by entanglement creation among the two-level qubit pair as long as the dipole-dipole frequency shift lies around the boson mode frequency. Generally, the entanglement effect enhances during the cooling process while the amplitude of the applied coherent field increases from zero. However, the maximum of the concurrence which was taken as entanglement measure, has slightly lower magnitudes than those that would be obtained but in the absence of the boson mode coupling to the two qubits. Thus, the maximum boson mode cooling efficiency realizes on the expense of the entanglement creation. Anyway, for lower bath temperatures, the concurrence will increase until values which also can be reached without the boson mode coupling to the qubit pair. An intuitive explanation for the described effects is as follows: Because we considered that the external coherent source frequency equals the qubit's transition frequency while its wave-vector is perpendicular to the line connecting the two dipole-dipole interacting qubits, then a privileged way to excite the collective sample is via a simultaneous absorption of a laser photon followed by a boson mode phonon absorption, respectively (see Fig. 1 1 ). This is because the dipole-dipole frequency splitting among the cooperative two-qubit states is close to the boson mode frequency. As a consequence, the qubit pair excitation as a whole results in the boson mode cooling, whereas the two closely spaced emitters get entangled. Finally, these effects occur for moderately weak external coherent driving fields which can avoid sample's deteriorations.

The article is organized as follows. In Sec. II we describe the system of interest together with the analytical approach used as well as the boson mode features. In Sec. III we analyze the entanglement creation among the qubit pair and its relationship with the boson mode cooling effects, respectively. We finalize the article with a summary given in Sec. IV. 
(a)

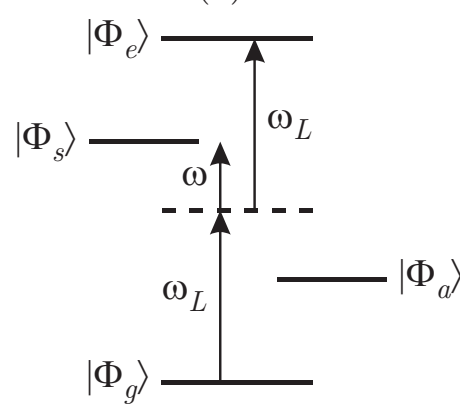

(b)

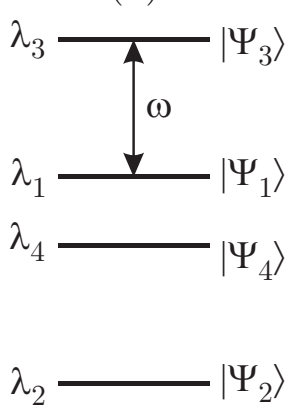

FIG. 1: (a) The two-qubit cooperative states $\left|\Phi_{i}\right\rangle$, where $i \in\{e, s, a, g\}$, stand for excited, symmetrical, antisymmetrical and ground Dicke states, respectively. A coherent laser source, with its wave-vector perpendicular to the line connecting the two qubits, can excite the whole sample either directly, i.e. $\left|\Phi_{g}\right\rangle \leftrightarrow\left|\Phi_{s}\right\rangle \leftrightarrow\left|\Phi_{e}\right\rangle$, or indirectly, that is $\left|\Phi_{g}\right\rangle \leftrightarrow\left|\Phi_{e}\right\rangle$. The latter path involves absorption of two photons simultaneously and is less probable for weaker applied external fields - a situation considered here. Therefore, the first channel prevalent meaning that qubit's subsystem excitation is taken place via absorption of a single laser photon of frequency $\omega_{L}$ followed, respectively, by a phonon absorption of frequency $\omega$. A process which leads to cooling of the single-mode boson field longitudinally coupled to the two qubits which become also entangled. (b) The two-qubit dressed-states $\left|\Psi_{j}\right\rangle$ and the corresponding eigenvalues $\lambda_{j}, j \in\{1,2,3,4\}$, obtained after diagonalization of the dipole-dipole coupled qubit-laser interaction Hamiltonian.

\section{THE MASTER EQUATION AND EQUATIONS OF MOTION}

The system of interest consists of environmental vacuum mediated dipole-dipole coupled pair of identical two-level $\left\{|2\rangle_{j},|1\rangle_{j}\right\}$ quantum emitters $\{j \in q 1, q 2\}$, resonantly pumped by a coherent laser source. The laser wavelength is sufficiently bigger than the interqubit spatial interval $\left|\vec{r}_{q_{1} q_{2}}\right|$, while the interparticle separation is larger than the linear size of the quantum emitter itself. Also, the laser wave-vector is perpendicular to the line connecting the qubits. Hence, the qubits are in an equivalent position with respect to the driving field. We have assumed that the transition frequencies of both qubits are equal and identical to the external pumping source frequency, respectively. Furthermore, the two-level qubits interact with a single boson mode of frequency $\omega$ via a longitudinal coupling. The whole system dampens via the interaction with the electromagnetic vacuum modes of the surrounding reservoir as well as through the corresponding boson mode environmental thermostat, respectively. As an 
appropriate realistic model can be taken a coherently pumped two-qubit pair, formed of ions, molecules, dimers or impurities as well as quantum dots or quantum wells, superconducting qubits etc., embedded in cavities, nanomechanical resonators or quantum circuits ones etc. [25, 40 53].

In the following, we shall present the corresponding master equation describing the investigated model where all the involved parameters are included properly.

\section{A. The master equation}

The master equation describing this global system in the Born-Markov approximations [1] 3, 9, 11] is given as follows

$$
\begin{aligned}
\dot{\rho} & +\frac{i}{\hbar}[\bar{H}, \rho]=-\sum_{\{j, l\} \in\{q 1, q 2\}}\left(\gamma_{j l}\left[S_{j}^{+}, S_{l}^{-} \rho\right]+H . c .\right) \\
& -\frac{\kappa}{2}(1+\bar{n})\left[b^{\dagger}, b \rho\right]-\frac{\kappa}{2} \bar{n}\left[b, b^{\dagger} \rho\right]+H . c .
\end{aligned}
$$

where an overdot denotes differentiation with respect to time. Here, the qubit operators $S_{j}^{+}=|2\rangle_{j j}\langle 1|, S_{j}^{-}=\left[S_{j}^{+}\right]^{\dagger}$ and $S_{z}^{(j)}=\left(|2\rangle_{j j}\langle 2|-| 1\rangle_{j j}\langle 1|\right) / 2$ obey the commutation relations: $\left[S_{j}^{+}, S_{l}^{-}\right]=2 S_{z}^{(j)} \delta_{j l}$ whereas $\left[S_{z}^{(j)}, S_{l}^{ \pm}\right]= \pm S_{j}^{ \pm} \delta_{j l}$. The respective boson mode creation, $b^{\dagger}$, and annihilation, $b$, operators satisfy the following commutation relations: $\left[b, b^{\dagger}\right]=1$ and $[b, b]=\left[b^{\dagger}, b^{\dagger}\right]=0$. In Eq. (1) , the Hamiltonian characterizing the corresponding coherent quantum dynamics is $\bar{H}=H+H_{i}$, where

$$
H=\hbar \omega b^{\dagger} b+\hbar g \sum_{j \in\{q 1, q 2\}} S_{z}^{(j)}\left(b+b^{\dagger}\right)
$$

and

$$
H_{i}=\hbar \Omega_{d d} \sum_{j \neq l \in\{q 1, q 2\}} S_{j}^{+} S_{l}^{-}+\hbar \Omega \sum_{j \in\{q 1, q 2\}}\left(S_{j}^{+}+S_{j}^{-}\right) .
$$

Here, $g$ is the qubit-boson-mode coupling strength whereas $\Omega$ denotes the standard Rabi frequency, both assumed to be identical for each qubit, respectively. $\gamma_{q 1 q 1}=\gamma_{q 2 q 2}=\gamma / 2$ is the single-qubit spontaneous decay rate, while $\gamma_{q 1 q 2}=\gamma_{q 2 q 1}=\gamma \chi_{r} / 2$ describes the radiative coupling among the two-level qubits and $\Omega_{d d}$ corresponds to the dipole-dipole interaction potential, respectively. The radiative coupling $\chi_{r}$ goes to zero (unity) for larger (smaller) 
interparticle separations $\left|\vec{r}_{q_{1} q_{2}}\right|$ in comparison to the photon emission wavelength. Correspondingly, $\Omega_{d d}$ tends to zero or to the static dipole-dipole interaction potential. Finally, $\kappa$ is the damping rate of the boson mode, while $\bar{n}$ gives its mean thermal phonon number corresponding to the frequency $\omega$ and environmental temperature $T$.

For our further purposes, we diagonalize the Hamiltonian (3) describing the dipole-dipole coupled qubit pair interacting as well with an externally applied coherent laser field, using the following two-qubit bare states: $\left|2_{q 1} 2_{q 2}\right\rangle,\left|2_{q 1} 1_{q 2}\right\rangle,\left|1_{q 1} 2_{q 2}\right\rangle$ and $\left|1_{q 1} 1_{q 2}\right\rangle$. Hence, we arrive at the corresponding cooperative two-qubit eigenfunctions:

$$
\begin{aligned}
\left|\Psi_{4}\right\rangle & =-\bar{a}\left\{\left|2_{q 1} 2_{q 2}\right\rangle+\left|1_{q 1} 1_{q 2}\right\rangle\right\}+\bar{b}\left\{\left|2_{q 1} 1_{q 2}\right\rangle+\left|1_{q 1} 2_{q 2}\right\rangle\right\} \\
\left|\Psi_{3}\right\rangle & =-\bar{c}\left\{\left|2_{q 1} 2_{q 2}\right\rangle+\left|1_{q 1} 1_{q 2}\right\rangle\right\}+\bar{d}\left\{\left|2_{q 1} 1_{q 2}\right\rangle+\left|1_{q 1} 2_{q 2}\right\rangle\right\} \\
\left|\Psi_{2}\right\rangle & =\frac{1}{\sqrt{2}}\left\{\left|2_{q 1} 1_{q 2}\right\rangle-\left|1_{q 1} 2_{q 2}\right\rangle\right\} \\
\left|\Psi_{1}\right\rangle & =\frac{1}{\sqrt{2}}\left\{\left|2_{q 1} 2_{q 2}\right\rangle-\left|1_{q 1} 1_{q 2}\right\rangle\right\}
\end{aligned}
$$

Here

$$
\begin{array}{ll}
\bar{a}=\frac{\left(\Omega_{d d}-\lambda_{4}\right) / \sqrt{2}}{\sqrt{\left(\Omega_{d d}-\lambda_{4}\right)^{2}+4 \Omega^{2}}}, & \bar{b}=\sqrt{\frac{2 \Omega^{2}}{\left(\Omega_{d d}-\lambda_{4}\right)^{2}+4 \Omega^{2}}}, \\
\bar{c}=\frac{\left(\Omega_{d d}-\lambda_{3}\right) / \sqrt{2}}{\sqrt{\left(\Omega_{d d}-\lambda_{3}\right)^{2}+4 \Omega^{2}}}, & \bar{d}=\sqrt{\frac{2 \Omega^{2}}{\left(\Omega_{d d}-\lambda_{3}\right)^{2}+4 \Omega^{2}}},
\end{array}
$$

with

$$
\begin{aligned}
& \lambda_{4}=\left(\Omega_{d d}-\sqrt{\Omega_{d d}^{2}+16 \Omega^{2}}\right) / 2, \\
& \lambda_{3}=\left(\Omega_{d d}+\sqrt{\Omega_{d d}^{2}+16 \Omega^{2}}\right) / 2,
\end{aligned}
$$

whereas other eigenvalues are

$$
\lambda_{2}=-\Omega_{d d}, \text { and } \lambda_{1}=0,
$$

respectively, see also Fig. 1(b). Substituting the two-qubit dressed-state transformation (4) in the master equation (11), while keeping the slowly varying terms only by assuming that $\omega>g$ with $\omega \approx \lambda_{3}$ as well as $\Omega_{d d} \gg \gamma$ and $\left|\lambda_{4}\right| \ll\left|\lambda_{2}\right|$, one arrives at the following main 
equation governing the quantum dynamics of the examined system

$$
\begin{aligned}
\dot{\rho}(t) & +\frac{i}{\hbar}\left[\bar{H}_{0}, \rho\right]=-\frac{\gamma}{2}\left(1+\chi_{r}\right)\left(2 \left[\bar{c} \bar{d} R_{44}+\bar{a} \bar{b} R_{33}+\frac{\bar{c}}{2 \sqrt{2}}\left(R_{41}-R_{14}\right),\left\{4\left(\bar{c} \bar{d} R_{44}+\bar{a} \bar{b} R_{33}\right)\right.\right.\right. \\
& \left.\left.+\sqrt{2} \bar{c}\left(R_{14}-R_{41}\right)\right\} \rho\right]+2(\bar{a} \bar{d}+\bar{b} \bar{c})^{2}\left\{\left[R_{34}, R_{43} \rho\right]+\left[R_{43}, R_{34} \rho\right]\right\}+\bar{a}^{2}\left\{\left[R_{13}, R_{31} \rho\right]\right. \\
& \left.\left.+\left[R_{31}, R_{13} \rho\right]\right\}-\sqrt{2} \bar{a}(\bar{a} \bar{d}+\bar{b} \bar{c})\left\{\left[R_{43}, R_{31} \rho\right]+\left[R_{13}, R_{34} \rho\right]-\left[R_{34}, R_{13} \rho\right]-\left[R_{31}, R_{43} \rho\right]\right\}\right) \\
& -\frac{\gamma}{2}\left(1-\chi_{r}\right)\left(\bar{b}^{2}\left\{\left[R_{32}, R_{23} \rho\right]+\left[R_{23}, R_{32} \rho\right]\right\}+\bar{d}^{2}\left\{\left[R_{24}, R_{42} \rho\right]+\left[R_{42}, R_{24} \rho\right]\right\}\right. \\
& +\frac{1}{2}\left\{\left[R_{12}, R_{21} \rho\right]+\left[R_{21}, R_{12} \rho\right]\right\}-\frac{\bar{d}}{\sqrt{2}}\left\{\left[R_{42}, R_{21} \rho\right]+\left[R_{12}, R_{24} \rho\right]-\left[R_{24}, R_{12} \rho\right]\right. \\
& \left.\left.-\left[R_{21}, R_{42} \rho\right]\right\}\right)-\frac{\kappa}{2}(1+\bar{n})\left[b^{\dagger}, b \rho\right]-\frac{\kappa}{2} \bar{n}\left[b, b^{\dagger} \rho\right]+H . c . .
\end{aligned}
$$

Here

$$
\bar{H}_{0}=\hbar \lambda_{4} R_{44}-\hbar \delta b^{\dagger} b-\hbar \bar{g}\left(R_{31} b+b^{\dagger} R_{13}\right)
$$

where $\delta=\lambda_{3}-\omega$, whereas $\bar{g}=\sqrt{2} g \bar{c}$, see also Fig. 1(b). The resulting two-qubit dressed-state operators which enter in Eq. (5) are defined as follows: $R_{\alpha \beta}=\left|\Psi_{\alpha}\right\rangle\left\langle\Psi_{\beta}\right|,\{\alpha, \beta \in 1, \cdots, 4\}$, and satisfy the standard commutation relations $\left[R_{\alpha \beta}, R_{\beta^{\prime} \alpha^{\prime}}\right]=R_{\alpha \alpha^{\prime}} \delta_{\beta \beta^{\prime}}-R_{\beta^{\prime} \beta} \delta_{\alpha^{\prime} \alpha}$. Note that $\left|\lambda_{4}\right| \ll\left|\lambda_{2}\right|$ means also that we deal with rather weaker applied laser fields, i.e. $\Omega / \Omega_{d d} \ll 1$ or the Rabi frequency $\Omega$ is of the order of few $\gamma$ 's or even less.

\section{B. The equations of motion}

Using the Master equation (5), one can obtain the following equations of motion describing the combined laser pumped qubit pair plus boson mode sample where the corresponding pumping and damping effects are properly taken into account:

$$
\begin{aligned}
\dot{P}_{n}^{(0)} & =i \bar{g}\left(P_{n}^{(4)}-P_{n}^{(6)}\right)-\kappa \bar{n}\left((n+1) P_{n}^{(0)}-n P_{n-1}^{(0)}\right) \\
& -\kappa(1+\bar{n})\left(n P_{n}^{(0)}-(n+1) P_{n+1}^{(0)}\right), \\
\dot{P}_{n}^{(1)} & =i \bar{g} P_{n}^{(4)}-\kappa \bar{n}\left((n+1) P_{n}^{(1)}-n P_{n-1}^{(1)}\right) \\
& -\kappa(1+\bar{n})\left(n P_{n}^{(1)}-(n+1) P_{n+1}^{(1)}\right)+\gamma_{0}^{(1)} P_{n}^{(0)} \\
& -\gamma_{1}^{(1)} P_{n}^{(1)}-\gamma_{2}^{(1)} P_{n}^{(2)}-\gamma_{3}^{(1)} P_{n}^{(3)}+\gamma_{11}^{(1)} P_{n}^{(11)},
\end{aligned}
$$




$$
\begin{aligned}
& \dot{P}_{n}^{(2)}=-\kappa(1+\bar{n})\left(n P_{n}^{(2)}-(n+1) P_{n+1}^{(2)}\right) \\
& -\kappa \bar{n}\left((n+1) P_{n}^{(2)}-n P_{n-1}^{(2)}\right)+\gamma_{0}^{(2)} P_{n}^{(0)} \\
& +\gamma_{1}^{(2)} P_{n}^{(1)}-\gamma_{2}^{(2)} P_{n}^{(2)}+\gamma_{3}^{(2)} P_{n}^{(3)}-\gamma_{11}^{(2)} P_{n}^{(11)}, \\
& \dot{P}_{n}^{(3)}=-i \bar{g} P_{n}^{(6)}+\gamma_{0}^{(3)} P_{n}^{(0)}+\gamma_{1}^{(3)} P_{n}^{(1)}-\gamma_{2}^{(3)} P_{n}^{(2)} \\
& -\gamma_{3}^{(3)} P_{n}^{(3)}-\gamma_{11}^{(3)} P_{n}^{(11)}-\kappa \bar{n}\left((n+1) P_{n}^{(3)}\right. \\
& \left.-n P_{n-1}^{(3)}\right)-\kappa(1+\bar{n})\left(n P_{n}^{(3)}-(n+1) P_{n+1}^{(3)}\right), \\
& \dot{P}_{n}^{(4)}=-i \delta P_{n}^{(5)}+2 i \bar{g} n\left(P_{n}^{(1)}-P_{n-1}^{(3)}\right)-\kappa(1+\bar{n})\left(2 P_{n}^{(6)}\right. \\
& \left.+(2 n-1) P_{n}^{(4)}-2(n+1) P_{n+1}^{(4)}\right) / 2+\kappa \bar{n}\left(2 n P_{n-1}^{(4)}\right. \\
& \left.-(2 n+1) P_{n}^{(4)}\right) / 2-\gamma_{4}^{(4)} P_{n}^{(4)}+\gamma_{8}^{(4)} P_{n}^{(8)}, \\
& \dot{P}_{n}^{(5)}=-i \delta P_{n}^{(4)}-\kappa(1+\bar{n})\left(2 P_{n}^{(7)}+(2 n-1) P_{n}^{(5)}\right. \\
& \left.-2(n+1) P_{n+1}^{(5)}\right) / 2-\kappa \bar{n}\left((2 n+1) P_{n}^{(5)}\right. \\
& \left.-2 n P_{n-1}^{(5)}\right) / 2-\gamma_{5}^{(5)} P_{n}^{(5)}+\gamma_{9}^{(5)} P_{n}^{(9)}, \\
& \dot{P}_{n}^{(6)}=-i \delta P_{n}^{(7)}+2 i \bar{g}(n+1)\left(P_{n+1}^{(1)}-P_{n}^{(3)}\right)-\kappa(1+\bar{n}) \\
& \times\left((2 n+1) P_{n}^{(6)}-2(n+1) P_{n+1}^{(6)}\right) / 2+\kappa \bar{n}\left(2 n P_{n-1}^{(6)}\right. \\
& \left.-(2 n+3) P_{n}^{(6)}+2 P_{n}^{(4)}\right) / 2-\gamma_{6}^{(6)} P_{n}^{(6)}+\gamma_{12}^{(6)} P_{n}^{(12)}, \\
& \dot{P}_{n}^{(7)}=-i \delta P_{n}^{(6)}-\kappa(1+\bar{n})\left((2 n+1) P_{n}^{(7)}-2(n+1)\right. \\
& \left.\times P_{n+1}^{(7)}\right) / 2-\kappa \bar{n}\left((2 n+3) P_{n}^{(7)}-2 n P_{n-1}^{(7)}\right. \\
& \left.-2 P_{n}^{(5)}\right) / 2-\gamma_{7}^{(7)} P_{n}^{(7)}+\gamma_{13}^{(7)} P_{n}^{(13)} \\
& \dot{P}_{n}^{(8)}=i\left(\lambda_{4}-\delta\right) P_{n}^{(9)}+i \bar{g} n P_{n}^{(11)}-\kappa \bar{n}\left((2 n+1) P_{n}^{(8)}\right. \\
& \left.-2 n P_{n-1}^{(8)}\right) / 2-\kappa(1+\bar{n})\left((2 n-1) P_{n}^{(8)}+2 P_{n}^{(12)}\right. \\
& \left.-2(n+1) P_{n+1}^{(8)}\right) / 2+\gamma_{4}^{(8)} P_{n}^{(4)}-\gamma_{8}^{(8)} P_{n}^{(8)}, \\
& \dot{P}_{n}^{(9)}=i\left(\lambda_{4}-\delta\right) P_{n}^{(8)}+i \bar{g} n P_{n}^{(10)}-\kappa \bar{n}\left((2 n+1) P_{n}^{(9)}\right. \\
& \left.-2 n P_{n-1}^{(9)}\right) / 2-\kappa(1+\bar{n})\left((2 n-1) P_{n}^{(9)}+2 P_{n}^{(13)}\right. \\
& \left.-2(n+1) P_{n+1}^{(9)}\right) / 2+\gamma_{5}^{(9)} P_{n}^{(5)}-\gamma_{9}^{(9)} P_{n}^{(9)}, \\
& \dot{P}_{n}^{(10)}=i \lambda_{4} P_{n}^{(11)}+i \bar{g} P_{n}^{(9)}-\kappa \bar{n}\left((n+1) P_{n}^{(10)}-n P_{n-1}^{(10)}\right) \\
& -\kappa(1+\bar{n})\left(n P_{n}^{(10)}-(n+1) P_{n+1}^{(10)}\right)-\gamma_{10}^{(10)} P_{n}^{(10)}, \\
& \dot{P}_{n}^{(11)}=i \lambda_{4} P_{n}^{(10)}+i \bar{g} P_{n}^{(8)}-\kappa \bar{n}\left((n+1) P_{n}^{(11)}-n P_{n-1}^{(11)}\right) \\
& -\kappa(1+\bar{n})\left(n P_{n}^{(11)}-(n+1) P_{n+1}^{(11)}\right)+\gamma_{0}^{(11)} P_{n}^{(0)}
\end{aligned}
$$



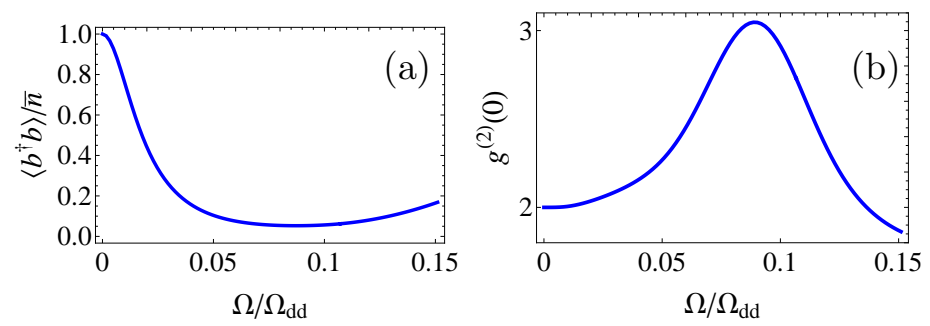

FIG. 2: (a) The steady-state behavior of the scaled mean phonon number $\left\langle b^{\dagger} b\right\rangle / \bar{n}$ as well as (b) the steady-state behavior of the second-order phonon-phonon correlation function $g^{(2)}(0)$ versus $\Omega / \Omega_{d d}$, respectively. The involved parameters are: $g / \gamma=2, \Omega_{d d} / \gamma=28, \omega / \gamma=30, \chi_{r}=0.98$, $\bar{n}=20$ and $\kappa / \gamma=10^{-3}$.

$$
\begin{aligned}
& -\gamma_{1}^{(11)} P_{n}^{(1)}-\gamma_{2}^{(11)} P_{n}^{(2)}-\gamma_{3}^{(11)} P_{n}^{(3)}-\gamma_{11}^{(11)} P_{n}^{(11)}, \\
\dot{P}_{n}^{(12)} & =i\left(\lambda_{4}-\delta\right) P_{n}^{(13)}+i \bar{g}(n+1) P_{n+1}^{(11)}+\gamma_{6}^{(12)} P_{n}^{(6)} \\
& -\kappa(1+\bar{n})\left((2 n+1) P_{n}^{(12)}-2(n+1) P_{n+1}^{(12)}\right) / 2 \\
& -\kappa \bar{n}\left((2 n+3) P_{n}^{(12)}-2 n P_{n-1}^{(12)}-2 P_{n}^{(8)}\right) / 2 \\
& -\gamma_{12}^{(12)} P_{n}^{(12)}, \\
\dot{P}_{n}^{(13)} & =i\left(\lambda_{4}-\delta\right) P_{n}^{(12)}+i \bar{g}(n+1) P_{n+1}^{(10)}+\gamma_{7}^{(13)} P_{n}^{(7)} \\
& -\kappa(1+\bar{n})\left((2 n+1) P_{n}^{(13)}-2(n+1) P_{n+1}^{(13)}\right) / 2 \\
& -\kappa \bar{n}\left((2 n+3) P_{n}^{(13)}-2 n P_{n-1}^{(13)}-2 P_{n}^{(9)}\right) / 2 \\
& -\gamma_{13}^{(13)} P_{n}^{(13)} .
\end{aligned}
$$

The system of equations (77) can be easily obtained if one first get the equations of motion for the variables $\rho_{\alpha \beta}=\langle\alpha|\rho| \beta\rangle,\{\alpha, \beta \in 1, \cdots, 4\}$, see also [54], using the Master Equation (5), namely, $\rho^{(0)}=\rho_{11}+\rho_{22}+\rho_{33}+\rho_{44}, \rho^{(1)}=\rho_{11}, \rho^{(2)}=\rho_{22}, \rho^{(3)}=\rho_{33}, \rho^{(4)}=b^{\dagger} \rho_{31}-\rho_{13} b$, $\rho^{(5)}=b^{\dagger} \rho_{31}+\rho_{13} b, \rho^{(6)}=\rho_{31} b^{\dagger}-b \rho_{13}, \rho^{(7)}=\rho_{31} b^{\dagger}+b \rho_{13}, \rho^{(8)}=b^{\dagger} \rho_{34}-\rho_{43} b, \rho^{(9)}=b^{\dagger} \rho_{34}+\rho_{43} b$, $\rho^{(10)}=\rho_{14}-\rho_{41}, \rho^{(11)}=\rho_{14}+\rho_{41}, \rho^{(12)}=\rho_{34} b^{\dagger}-b \rho_{43}$, and $\rho^{(13)}=\rho_{34} b^{\dagger}+b \rho_{43}$. The projection on the Fock states $|n\rangle$, i.e., $P_{n}^{(i)}=\left\langle n\left|\rho^{(i)}\right| n\right\rangle,\{i \in 0, \cdots, 13\}$, with $n \in\{0, \infty\}$, will lead us to Eqs. (77). The corresponding decay rates are given in the Appendix A.

Generally, in order to solve the infinite system of equations (7), one truncates it at a certain maximum value $n=n_{\max }$ so that a further increase of its value, i.e. $n_{\max }$, does not modify the obtained results. As a consequence, the steady-state mean phonon number is 
expressed as:

$$
\left\langle b^{\dagger} b\right\rangle=\sum_{n=0}^{n_{\max }} n P_{n}^{(0)},
$$

with

$$
\sum_{n=0}^{n_{\max }} P_{n}^{(0)}=1
$$

while its steady-state second-order phonon-phonon correlation function is defined in the usual way [55], namely,

$$
\begin{aligned}
g_{b}^{(2)}(0) & =\frac{\left\langle b^{\dagger} b^{\dagger} b b\right\rangle}{\left\langle b^{\dagger} b\right\rangle^{2}} \\
& =\frac{1}{\left\langle b^{\dagger} b\right\rangle^{2}} \sum_{n=0}^{n_{\max }} n(n-1) P_{n}^{(0)} .
\end{aligned}
$$

Based on Eqs. (7] $8[9][10)$, Figure 2(a) shows the cooling of the boson mode while the pumping parameter is being varying demonstrating an efficient cooling scheme. Respectively, Figure 2(b) depicts the second-order phonon-phonon correlation function, during the cooling process, demonstrating super-Poissonian phonon statistics, i.e. $g^{(2)}(0)>2$, with only few phonons. The cooling mechanism occurring in this system, when $\Omega \ll \Omega_{d d}$, can be intuitively understood if one refers to the two-qubit Dicke states [1-3], namely, $\left|\Phi_{e}\right\rangle=\left|2_{q 1} 2_{q^{2}}\right\rangle$, $\left|\Phi_{s}\right\rangle=\left\{\left|2_{q 1} 1_{q 2}\right\rangle+\left|1_{q 1} 2_{q 2}\right\rangle\right\} / \sqrt{2},\left|\Phi_{a}\right\rangle=\left\{\left|2_{q 1} 1_{q 2}\right\rangle-\left|1_{q 1} 2_{q 2}\right\rangle\right\} / \sqrt{2}$, and $\left|\Phi_{g}\right\rangle=\left|1_{q 1} 1_{q 2}\right\rangle$, see Fig. 1(a). When the external field frequency is in resonance with that of the qubit one, while its wave-vector is perpendicular to the line connecting the two qubits, then the only way to laser excite the two-qubit sample is either via $\left|\Phi_{g}\right\rangle \rightarrow\left|\Phi_{s}\right\rangle \rightarrow\left|\Phi_{e}\right\rangle$ or $\left|\Phi_{g}\right\rangle \rightarrow\left|\Phi_{e}\right\rangle$, respectively. The latter path involve two-photon processes which are less probable for weaker external driving fields. Therefore, the first channel, i.e. $\left|\Phi_{g}\right\rangle \rightarrow\left|\Phi_{s}\right\rangle \rightarrow\left|\Phi_{e}\right\rangle$, becomes active and involves an available phonon at a particular frequency from simple reasons since the driving external coherent field is in resonance with the qubit's transition frequency, that is $\omega_{s g} \approx \omega_{L}+\omega$, see Fig. 1(a). Thus, one can conclude that the two-qubit system absorbs an external laser photon followed by a boson mode phonon absorption in order to reach the symmetrical Dicke state $\left|\Phi_{s}\right\rangle$, and these processes lead to phonon cooling effects, respectively. Figure 3(a) demonstrates this statement in the sense that the population of the symmetrical two-qubit state, i.e. $\left|\Phi_{s}\right\rangle$, increases in the presence of the boson mode, coupled to the two qubits, while compared to the case of its absence. In the dressed-state picture, 

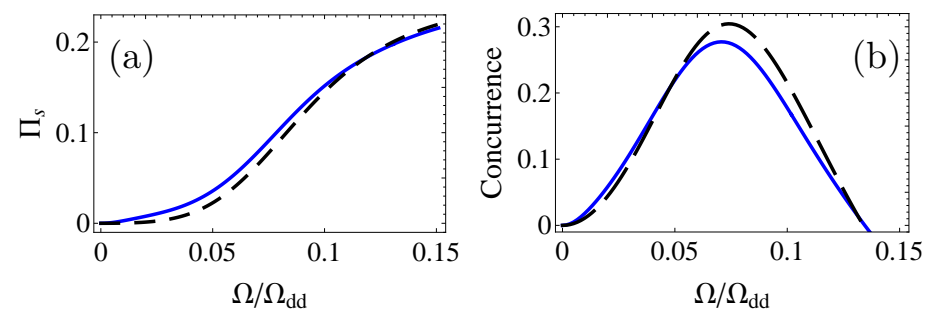

FIG. 3: (a) The steady-state behaviors of the population in the symmetrical two-qubit Dicke state $\left|\Phi_{s}\right\rangle=\left\{\left|2_{q 1} 1_{q 2}\right\rangle+\left|1_{q 1} 2_{q 2}\right\rangle\right\} / \sqrt{2}$, i.e. $\Pi_{s}=\left\langle\mid \Phi_{s}\right\rangle\left\langle\Phi_{s} \mid\right\rangle$, as a function of scaled pumping parameter $\Omega / \Omega_{d d}$. (b) The corresponding behaviors but for the two-qubit concurrence $C$. In these plots the solid lines are plotted for $g \neq 0$ while the dashed curves describe the same results but with $g=0$, respectively. All other parameters are the same as for Fig. (2).

depicted in Fig. 1(b), cooling occurs evidently when the population in the dressed-state $\left|\Psi_{1}\right\rangle$ is larger than that residing in the state $\left|\Psi_{3}\right\rangle$, respectively. If one inspects the explicit forms of these states, see Exps. (4), then one can observe that laser excitation involves directly all the two-qubit Dicke states, excepting the antisymmetrical one, i.e. $\left|\Phi_{a}\right\rangle$.

Until now we have focused mainly on the boson mode properties. In the following Section using the analytical approach developed here, we shall investigate the entanglement creation within the qubit subsystem and emphasize its connection to the boson mode cooling phenomena, respectively.

\section{ENTANGLEMENT OF THE TWO-QUBIT SYSTEM COUPLED WITH A SINGLE-MODE BOSON FIELD}

The entanglement and its definition is certainly a main topic within quantum computation theory. In this context, one of the widely accepted measures of entanglement for a two qubit system is the concurrence, $C$, [4, 5]. Particularly, for a mixed state of qubits $\left\{q_{1}, q_{2}\right\}$ with density matrix $\tilde{\rho}_{q_{1} q_{2}}$, it is defined as

$$
C=\max \left\{0, \mathrm{~s}_{1}-\sum_{\xi=2}^{4} \mathrm{~s}_{\xi}\right\} .
$$

The quantities $s_{\xi},\{\xi \in 1, \cdots, 4\}$, are the square roots of the eigenvalues of the following matrix product

$$
Q=\tilde{\rho}_{q_{1} q_{2}}\left(\sigma_{q_{1} y} \otimes \sigma_{q_{2} y}\right) \tilde{\rho}_{q_{1} q_{2}}^{*}\left(\sigma_{q_{1} y} \otimes \sigma_{q_{2} y}\right)
$$


and, importantly, in descending order. Here, $\tilde{\rho}_{q_{1} q_{2}}^{*}$ denotes complex conjugation of $\tilde{\rho}_{q_{1} q_{2}}$, and $\sigma_{j y}$ are Pauli matrices for the two-level systems $\left(j \in\left\{q_{1}, q_{2}\right\}\right)$. The values of the concurrence range from zero for an unentangled state to unity for a maximally entangled two-particle state [4, 5]. The density matrix $\tilde{\rho}_{q_{1} q_{2}}$ can be represented in the basis $\left|2_{q 1} 2_{a 2}\right\rangle,\left|2_{a 1} 1_{a 2}\right\rangle,\left|1_{q 1} 2_{q 2}\right\rangle$ and $\left|1_{q 1} 1_{q 2}\right\rangle$, which is symmetric under the exchange of the sub-systems [4, 5, 14, 16]. Hence, its elements are given as follows:

$$
\tilde{\rho}_{q_{1} q_{2}}=\left(\begin{array}{cccc}
\tilde{\rho}_{11} & \tilde{\rho}_{12} & \tilde{\rho}_{13} & \tilde{\rho}_{14} \\
\tilde{\rho}_{21} & \tilde{\rho}_{22} & \tilde{\rho}_{23} & \tilde{\rho}_{24} \\
\tilde{\rho}_{31} & \tilde{\rho}_{32} & \tilde{\rho}_{33} & \tilde{\rho}_{34} \\
\tilde{\rho}_{41} & \tilde{\rho}_{42} & \tilde{\rho}_{43} & \tilde{\rho}_{44}
\end{array}\right),
$$

where

$$
\begin{aligned}
\tilde{\rho}_{11} & =\frac{1}{4}\left(1+\rho_{11}-\rho_{22}\right)-\frac{\Omega_{d d}}{4 \sqrt{\Omega_{d d}^{2}+(4 \Omega)^{2}}}\left(\rho_{33}-\rho_{44}\right) \\
& -\frac{\bar{a}}{\sqrt{2}}\left(\rho_{41}+\rho_{14}\right), \\
\tilde{\rho}_{12} & =\frac{\Omega}{\sqrt{\Omega_{d d}^{2}+(4 \Omega)^{2}}}\left(\rho_{33}-\rho_{44}\right)-\frac{\bar{c}}{\sqrt{2}} \rho_{41}, \\
\tilde{\rho}_{13} & =\tilde{\rho}_{12}, \\
\tilde{\rho}_{14} & =\frac{1}{4}\left(1+\frac{\Omega_{d d}}{\sqrt{\Omega_{d d}^{2}+(4 \Omega)^{2}}}\right) \rho_{44}-\frac{\bar{a}}{\sqrt{2}}\left(\rho_{41}-\rho_{14}\right) \\
& +\frac{1}{4}\left(1-\frac{\Omega_{d d}}{\sqrt{\Omega_{d d}^{2}+(4 \Omega)^{2}}}\right) \rho_{33}-\frac{1}{2} \rho_{11}, \\
\tilde{\rho}_{21} & =\left(\tilde{\rho}_{12}\right)^{\dagger}, \quad \Omega_{d d} \\
\tilde{\rho}_{22} & =\frac{1}{4}\left(1+\rho_{22}-\rho_{11}\right)+\frac{\Omega_{d d}}{4 \sqrt{\Omega^{2}+(4 \Omega)^{2}}}\left(\rho_{33}-\rho_{44}\right), \\
\tilde{\rho}_{23} & =\frac{1}{4}\left(1-\frac{1}{\sqrt{\Omega_{d d}^{2}+(4 \Omega)^{2}}}\right) \rho_{44}-\frac{1}{2} \rho_{22} \\
& +\frac{1}{4}\left(1+\frac{\Omega_{d d}}{\sqrt{\Omega_{d d}^{2}+(4 \Omega)^{2}}}\right) \rho_{33}, \\
\tilde{\rho}_{24} & =\frac{\tilde{\rho}_{31}}{\sqrt{\Omega_{d d}^{2}+(4 \Omega)^{2}}\left(\rho_{33}-\rho_{44}\right)+\frac{\bar{c}}{\sqrt{2}} \rho_{14},} \\
\tilde{\rho}_{41} & =\left(\tilde{\rho}_{13}\right)^{\dagger}, \quad \tilde{\rho}_{32}=\tilde{\rho}_{23}, \quad \tilde{\rho}_{33}=\tilde{\rho}_{22}, \quad \tilde{\rho}_{34}=\left(\tilde{\rho}_{24}\right)^{\dagger}, \quad \tilde{\rho}_{43}=\left(\tilde{\rho}_{34}\right)^{\dagger},
\end{aligned}
$$




$$
\begin{aligned}
\tilde{\rho}_{44} & =\frac{1}{4}\left(1+\rho_{11}-\rho_{22}\right)-\frac{\Omega_{d d}}{4 \sqrt{\Omega_{d d}^{2}+(4 \Omega)^{2}}}\left(\rho_{33}-\rho_{44}\right) \\
& +\frac{\bar{a}}{\sqrt{2}}\left(\rho_{41}+\rho_{14}\right) .
\end{aligned}
$$

Inserting the matrix (13) in the expression (12) one can obtain the corresponding eigenvalues of $Q$ after some algebraic manipulations. The corresponding steady-state behaviors for the concurrence $C$ are shown in the Figure 3(b) which were obtained with the help of Eqs. (7) as well as Exps. (11,14). One can observe that the entanglement creation among the twolevel qubit pair is accompanied by cooling of the boson mode (compare the solid curves in Fig. 2(a) and Fig. 3(b), respectively). Moreover, the maximal cooling effect is achieved when the entanglement is maximal as well. Note that even higher magnitudes for the concurrence $C$ can be obtained but in the absence of the qubit's coupling to the single-mode boson field (compare the solid and dashed curves in Fig. 3b) meaning that the maximal cooling efficiency realizes on the expense of the entanglement creation. Anyway, at the beginning of the steady-state evolution the concurrence $C$ is larger than its value which would be obtained, however, in the absence of the boson mode, see Fig. 3(b). Furthermore, for lower bath temperatures, the magnitude of the concurrence $C$ will reach the same values regardless of the boson mode presence. This is because at those temperatures the corresponding twoqubit cooperative states, responsible for entanglement creation, are almost equal populated in both cases.

\section{SUMMARY}

Summarizing, we have investigated the relationship among the entanglement creation in a laser-pumped dipole-dipole interacting two-level qubits and the cooling effects of a boson mode which is longitudinally coupled with the both quantum emitters, respectively. We have found that cooling occurs when the dipole-dipole frequency shift lies around the boson mode frequency. This happens because it was assumed that the driving coherent field frequency is equal with the qubit's transition one, while the two qubits are in an equivalent position with respect to the pumping external field. Hence, the only way to excite the two-qubit sample is via a concomitant absorption of a photon and a phonon, respectively, leading to cooling of the boson mode. Furthermore, the quantum cooling process is accompanied by entanglement creation within the qubit sample which is demonstrated by nonzero values 
for the concurrence, although its maximal magnitude, i.e. for $C$, is lower than that which would be obtained but in the absence of the single-mode boson field. However, adjusting the external parameters one can optimize the entanglement as well. These effects are taken place for rather weak external applied fields which may protect the sample from deteriorations.

This work was supported by grant No. 15.817.02.09F. Also, M.M.A. is grateful for the nice hospitality of the Theory Department of the Horia Hulubei National Institute of Physics and Nuclear Engineering, Bucharest, Romania.

\section{Appendix A: The decay rates entering in the equations of motion (7)}

Below one can find the corresponding decay rates which enter in the Eqs. (77), that is, $\gamma_{0}^{(1)}=\gamma \bar{c}^{2}\left(1+\chi_{r}\right), \gamma_{1}^{(1)}=\gamma\left\{\left(\bar{a}^{2}+2 \bar{c}^{2}\right)\left(1+\chi_{r}\right)+\left(1-\chi_{r}\right) / 2\right\}, \gamma_{2}^{(1)}=\gamma\left\{\bar{c}^{2}\left(1+\chi_{r}\right)-\left(1-\chi_{r}\right) / 2\right\}$, $\gamma_{3}^{(1)}=\gamma\left(1+\chi_{r}\right)\left(\bar{c}^{2}-\bar{a}^{2}\right), \gamma_{11}^{(1)}=\gamma\left\{\left(1+\chi_{r}\right)\left(\bar{a}(\bar{a} \bar{d}+\bar{b} \bar{c}) / \sqrt{2}+\sqrt{2} \bar{d} \bar{c}^{2}\right)+\frac{\bar{d}}{2 \sqrt{2}}\left(1-\chi_{r}\right)\right\}, \gamma_{0}^{(2)}=$ $\gamma \bar{d}^{2}\left(1-\chi_{r}\right), \gamma_{1}^{(2)}=\gamma\left(1-\chi_{r}\right)\left(1 / 2-\bar{d}^{2}\right), \gamma_{2}^{(2)}=\gamma\left(1-\chi_{r}\right)\left(1 / 2+\bar{b}^{2}+2 \bar{d}^{2}\right), \gamma_{3}^{(2)}=\gamma\left(1-\chi_{r}\right)\left(\bar{b}^{2}-\bar{d}^{2}\right)$, $\gamma_{11}^{(2)}=\gamma \bar{d}\left(1-\chi_{r}\right) / \sqrt{2}, \gamma_{0}^{(3)}=2 \gamma(\bar{a} \bar{d}+\bar{b} \bar{c})^{2}\left(1+\chi_{r}\right), \gamma_{1}^{(3)}=\gamma\left\{\bar{a}^{2}-2(\bar{a} \bar{d}+\bar{b} \bar{c})^{2}\right\}\left(1+\chi_{r}\right), \gamma_{2}^{(3)}=$ $\gamma\left\{2(\bar{a} \bar{d}+\bar{b} \bar{c})^{2}\left(1+\chi_{r}\right)-\bar{b}^{2}\left(1-\chi_{r}\right)\right\}, \gamma_{3}^{(3)}=2 \gamma\left\{\left(2(\bar{a} \bar{d}+\bar{b} \bar{c})^{2}+\bar{a}^{2} / 2\right)\left(1+\chi_{r}\right)+\bar{b}^{2}\left(1-\chi_{r}\right) / 2\right\}, \gamma_{11}^{(3)}=$ $\sqrt{2} \bar{a} \gamma(\bar{a} \bar{d}+\bar{b} \bar{c})\left(1+\chi_{r}\right), \gamma_{4}^{(4)}=\gamma\left\{\left(4(\bar{a} \bar{b})^{2}+(\bar{a} \bar{d}+\bar{b} \bar{c})^{2}+\bar{a}^{2}+\bar{c}^{2} / 2\right)\left(1+\chi_{r}\right)+\left(1 / 2+\bar{b}^{2}\right)\left(1-\chi_{r}\right) / 2\right\}$, $\gamma_{8}^{(4)}=\gamma\left\{(\sqrt{2} \bar{c}(2 \bar{a} \bar{b}+\bar{c} \bar{d})+\bar{a}(\bar{a} \bar{d}+\bar{b} \bar{c}) / \sqrt{2})\left(1+\chi_{r}\right)+\frac{\bar{d}}{2 \sqrt{2}}\left(1-\chi_{r}\right)\right\}, \gamma_{5}^{(5)}=\gamma_{4}^{(4)}, \gamma_{9}^{(5)}=\gamma_{8}^{(4)}$, $\gamma_{6}^{(6)}=\gamma_{5}^{(5)}, \gamma_{12}^{(6)}=\gamma_{9}^{(5)}, \gamma_{7}^{(7)}=\gamma_{6}^{(6)}, \gamma_{13}^{(7)}=\gamma_{12}^{(6)}, \gamma_{4}^{(8)}=\gamma\{(\sqrt{2} \bar{c}(\bar{c} \bar{d}-2 \bar{a} \bar{b})+\bar{a}(\bar{a} \bar{d}+\bar{b} \bar{c}) / \sqrt{2})(1+$ $\left.\left.\chi_{r}\right)+\frac{\bar{d}}{2 \sqrt{2}}\left(1-\chi_{r}\right)\right\}, \gamma_{8}^{(8)}=\gamma\left\{\left(4(\bar{a} \bar{b}-\bar{c} \bar{d})^{2}+2(\bar{a} \bar{d}+\bar{b} \bar{c})^{2}+\bar{a}^{2} / 2+\bar{c}^{2} / 2\right)\left(1+\chi_{r}\right)+\left(\bar{d}^{2}+\bar{b}^{2}\right)\left(1-\chi_{r}\right) / 2\right\}$, $\gamma_{5}^{(9)}=\gamma_{4}^{(8)}, \gamma_{9}^{(9)}=\gamma_{8}^{(8)}, \gamma_{10}^{(10)}=\gamma\left\{\left(4(\bar{c} \bar{d})^{2}+(\bar{a} \bar{d}+\bar{b} \bar{c})^{2}+\bar{a}^{2} / 2\right)\left(1+\chi_{r}\right)+\left(1 / 2+\bar{d}^{2}\right)\left(1-\chi_{r}\right) / 2\right\}$, $\gamma_{0}^{(11)}=2 \gamma\left\{\left(3 \sqrt{2} \bar{d} \bar{c}^{2}+\bar{a}(\bar{a} \bar{d}+\bar{b} \bar{c}) / \sqrt{2}\right)\left(1+\chi_{r}\right)+\frac{\bar{d}}{2 \sqrt{2}}\left(1-\chi_{r}\right)\right\}, \gamma_{1}^{(11)}=2 \sqrt{2} \gamma \bar{d} \bar{c}^{2}\left(1+\chi_{r}\right), \gamma_{2}^{(11)}=$ $2 \gamma\left\{\left(3 \sqrt{2} \bar{d} \bar{c}^{2}+\bar{a}(\bar{a} \bar{d}+\bar{b} \bar{c}) / \sqrt{2}\right)\left(1+\chi_{r}\right)-\frac{\bar{d}}{2 \sqrt{2}}\left(1-\chi_{r}\right)\right\}, \gamma_{3}^{(11)}=2 \gamma\left\{\left(3 \sqrt{2} \bar{d} \bar{c}^{2}-\bar{a}(\bar{a} \bar{d}+\bar{b} \bar{c}) / \sqrt{2}\right)(1+\right.$ $\left.\left.\chi_{r}\right)+\frac{\bar{d}}{2 \sqrt{2}}\left(1-\chi_{r}\right)\right\}, \gamma_{11}^{(11)}=\gamma\left\{\left(4(\bar{c} \bar{d})^{2}+(\bar{a} \bar{d}+\bar{b} \bar{c})^{2}+\bar{a}^{2} / 2+2 \bar{c}^{2}\right)\left(1+\chi_{r}\right)+\left(1 / 2+\bar{d}^{2}\right)\left(1-\chi_{r}\right) / 2\right\}$, $\gamma_{6}^{(12)}=\gamma_{7}^{(13)}=\gamma_{4}^{(8)}, \gamma_{12}^{(12)}=\gamma_{13}^{(13)}=\gamma_{8}^{(8)}$.

[1] R. H. Lehmberg, Radiation from an N-atom system. I. General formalism, Phys. Rev. A 2, $883(1970)$.

[2] R. H. Lehmberg, Radiation from an N-atom system. II. Spontaneous emission from a pair of atoms, Phys. Rev. A 2, 889 (1970). 
[3] Z. Ficek and S. Swain, Quantum Interference and Coherence: Theory and Experiments (Springer, Berlin, 2005).

[4] S. Hill, and W. K. Wootters, Entanglement of a pair of quantum bits, Phys. Rev. Lett. 78, 5022 (1997).

[5] W. K. Wootters, Entanglement of formation of an arbitrary state of two qubits, Phys. Rev. Lett. 80, 2245 (1998).

[6] M. D. Lukin and P. R. Hemmer, Quantum entanglement via optical control of atom-atom interactions, Phys. Rev. Lett. 84, 2818 (2000).

[7] T. Yu and J. H. Eberly, Finite-time disentanglement via spontaneous emission, Phys. Rev. Lett. 93, 140404 (2004).

[8] F. Tacchino, A. Aufféves, M. F. Santos and D. Gerace, Steady State Entanglement beyond Thermal Limits, Phys. Rev. Lett. 120, 063604 (2018).

[9] M. Scully, M. S. Zubairy, Quantum Optics (Cambridge University Press, Cambridge, 1997).

[10] M. A. Nielsen and I. L. Chuang, Quantum Computation and Quantum Information (Cambridge University Press, Cambridge, 2000).

[11] G. S. Agarwal, Quantum Optics (Cambridge University Press, 2014).

[12] J. I. Cirac, P. Zoller, H. J. Kimble, H. Mabuchi, Quantum state transfer and entanglement distribution among distant nodes in a quantum network, Phys. Rev. Lett. 78, 3221 (1997).

[13] C. H. Keitel, Narrowing spontaneous emission without intensity reduction, Phys. Rev. Lett. 83, 1307 (1999).

[14] X. Wang and K. Molmer, Pairwise entanglement in symmetric multi-qubit systems, Eur. Phys. J. D 18, 385 (2002).

[15] H. J. Kimble, The quantum internet, Nature 453, 1023 (2008).

[16] M. Macovei, J. Evers and C. H. Keitel, Quantum entanglement in dense multiqubit systems, J. Mod. Opt. 57, 1287 (2010).

[17] C. Ates, J. P. Garrahan, and I. Lesanovsky, Thermalization of a Strongly Interacting Closed Spin System: From Coherent Many-Body Dynamics to a Fokker-Planck Equation, Phys. Rev. Lett. 108, 110603 (2012).

[18] A. Streltsov, G. Adesso and M. B. Plenio, Colloquium: Quantum coherence as a resource, Rev. Mod. Phys. 89, 041003 (2017).

[19] L. Pezze, A. Smerzi, M. K. Oberthaler, R. Schmied and P. Treutlein, Quantum metrology 
with nonclassical states of atomic ensembles, Rev. Mod. Phys. 90, 035005 (2018).

[20] Z. Ficek, R. Tanas, Entangled states and collective nonclassical effects in two-atom systems, Phys. Rep. 372, 369 (2002).

[21] Yu. A. Pashkin, T. Yamamoto, O. Astafiev, Y. Nakamura, D. V. Averin and J. S. Tsai, Quantum oscillations in two coupled charge qubits, Nature 421, 823 (2003).

[22] G.-x. Li, Y.-p. Yang, K. Allaart, and D. Lenstra, Entanglement for excitons in two quantum dots in a cavity injected with squeezed vacuum, Phys. Rev. A 69, 014301 (2004).

[23] K. Xia, M. Macovei and J. Evers, Stationary entanglement in strongly coupled qubits, Phys. Rev. B 84, 184510 (2011).

[24] E. Cecoi, V. Ciornea, A. Isar and M. A. Macovei, Entanglement of a laser-driven pair of two-level qubits via its phonon environment, J. Opt. Soc. Am. B 35, 1127 (2018).

[25] A. Delteil, Z. Sun, S. Fält and A. Imamoğlu, Realization of a Cascaded Quantum System: Heralded Absorption of a Single Photon Qubit by a Single-Electron Charged Quantum Dot, Phys. Rev. Lett. 118, 177401 (2017).

[26] M. A. Macovei, Cooling a quantum circuit via coupling to a multiqubit system, Phys. Rev. A 81, $043411(2010)$.

[27] K. Xia and J. Evers, Ground-state cooling of a nanomechanical resonator coupled to two interacting flux qubits, Phys. Rev. B 82, 184532 (2010).

[28] G.-x. Li and J.-p. Zhu, Ground-state cooling of a mechanical resonator coupled to two coupled quantum dots, J. Phys. B: At. Mol. Opt. Phys. 44, 195502 (2011).

[29] V. Montenegro, R. Coto, V. Eremeev and M. Orszag, Ground-state cooling of a nanomechanical oscillator with $N$ spins, Phys. Rev. A 98, 053837 (2018).

[30] N. Brunner, M. Huber, N. Linden, S. Popescu, R. Silva, P. Skrzypczyk, Entanglement enhances cooling in microscopic quantum refrigerators, Phys. Rev. E 89, 032115 (2014).

[31] C. Genes, H. Ritsch, M. Drewsen, A. Dantan, Atom-membrane cooling and entanglement using cavity electromagnetically induced transparency, Phys. Rev. A 84, 051801(R) (2011).

[32] C. L. Cortes, M. Otten and S. K. Gray, Ground-state cooling enabled by critical coupling and dark entangled states, Phys. Rev. B 99, 014107 (2019).

[33] S. O. Valenzuela, W. D. Oliver, D. M. Berns, K. K. Berggren, L. S. Levitov and T. P. Orlando, Microwave-Induced Cooling of a Superconducting Qubit, Science 314, 1589 (2006).

[34] P. Marian and T. A. Marian, Entanglement of Formation for an Arbitrary Two-Mode Gaussian 
State, Phys. Rev. Lett. 101, 220403 (2008).

[35] C. Genes, D. Vitali, P. Tombesi, Simultaneous cooling and entanglement of mechanical modes of a micromirror in an optical cavity, New Journal of Physics 10, 095009 (2008).

[36] G. Vacanti and A. Beige, Cooling atoms into entangled states, New J. Phys. 11, 083008 (2009).

[37] N. Linden, S. Popescu and P. Skrzypczyk, How Small Can Thermal Machines Be? The Smallest Possible Refrigerator, Phys. Rev. Lett. 105, 130401 (2010).

[38] Z. Wang, W. Wu and J. Wang, Steady-state entanglement and coherence of two coupled qubits in equilibrium and nonequilibrium environments, Phys. Rev. A 99, 042320 (2019).

[39] R. Grimaudo, A. Isar, T. Mihaescu, I. Ghiu, A. Messina, Dynamics of quantum discord of two coupled spin- $1 / 2$ 's subjected to time-dependent magnetic fields, Results in Physics 13, 102147 (2019).

[40] R. Blatt, D. Wineland, Entangled states of trapped atomic ions, Nature 453, 1008 (2008).

[41] S. Genway, W. Li, C. Ates, B. P. Lenyon amd I. Lesanovsky, Generalized Dicke Nonequilibrium Dynamics in Trapped Ions, Phys. Rev. Lett. 112, 023603 (2014).

[42] P. Kirton and J. Keeling, Nonequilibrium Model of Photon Condensation, Phys. Rev. Lett. 111, $100404(2013)$

[43] S. F. Huelga, A. Rivas and M. B. Plenio, Non-Markovianity-Assisted Steady State Entanglement, Phys. Rev. Lett. 108, 160402 (2012).

[44] I. Martin, A. Shnirman, L. Tian and P. Zoller, Ground-state cooling of mechanical resonators, Phys. Rev. B 69, 125339 (2004).

[45] Y. Makhlin, G. Schön, A. Shnirman, Quantum-state engineering with Josephson-junction devices, Rev. Mod. Phys. 73, 357 (2001).

[46] S. Andre, V. Brosco, M. Marthaler, A. Shnirman and G. Schön, Few-qubit lasing in circuit QED, Phys. Scr. T137, 014016 (2009).

[47] J.-M. Pirkkalainen, S. U. Cho, J. Li, G. S. Paraoanu, P. J. Hakonen and M. A. Sillanpää, Hybrid circuit cavity quantumelectrodynamics with a micromechanical resonator, Nature 494, $211(2013)$.

[48] J. A. Mlynek, A. A. Abdumalikov, C. Eichler and A. Wallraff, Observation of Dicke superradiance for two artificial atoms in a cavity with high decay rate, Nat. Commun. 5:5186 (2014).

[49] D. Hucul, I. V. Inlek, G. Vittorini, C. Crocker, S. Debnath, S. M. Clark and C. Monroe, 
Modular entanglement of atomic qubits using photons and phonons, Nat. Phys. 11, 37 (2015).

[50] F. Armata, G. Calajo, T. Jaako, M. S. Kim and P. Rabl, Harvesting Multiqubit Entanglement from Ultrastrong Interactions in Circuit Quantum Electrodynamics, Phys. Rev. Lett. 119, $183602(2017)$.

[51] J. Wang, S. Paesani, Y. Ding, R. Santagati, P. Skrzypczyk, A. Salavrakos, J. Tura, R. Augusiak, L. Mancinska, D. Bacco, D. Bonneau, J. W. Silverstone, Q. Gong, A. Acn, K. Rottwitt, L. K. Oxenlowe, J. L. O'Brien, A. Laing and M. G. Thompson, Multidimensional quantum entanglement with large-scale integrated optics, Science 360, 285 (2018).

[52] M.-L. Hua, X. Huc, J. Wanga, Y. Peng, Y.-R. Zhang and H. Fan, Quantum coherence and geometric quantum discord, Phys. Rep. 762-764, 1 (2018).

[53] A. F. Kockum, A. Miranowicz, S. D. Liberato, S. Savasta and F. Nori, Ultrastrong coupling between light and matter, Nat. Rev. Phys. 1, 19 (2019).

[54] T. Quang and H. Freedhoff, Atomic population inversion and enhancement of resonance fluorescence in a cavity, Phys. Rev. A 47, 2285 (1993).

[55] R. J. Glauber, The Quantum Theory of Optical Coherence, Phys. Rev. 130, 2529 (1963). 